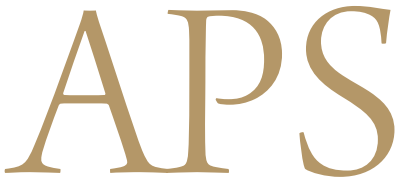

Archives of Plastic Surgery

\title{
Orthodromic Transfer of the Temporalis Muscle in Incomplete Facial Nerve Palsy
}

\author{
Jae Ho Aum, Dong Hee Kang, Sang Ah Oh, Ja Hea Gu \\ Department of Plastic and Reconstructive Surgery, Dankook University Hospital, Dankook University College of Medicine, Cheonan, Korea
}

Background Temporalis muscle transfer produces prompt surgical results with a one-stage operation in facial palsy patients. The orthodromic method is surgically simple, and the vector of muscle action is similar to the temporalis muscle action direction. This article describes transferring temporalis muscle insertion to reconstruct incomplete facial nerve palsy patients. Methods Between August 2009 and November 2011, 6 unilateral incomplete facial nerve palsy patients underwent surgery for orthodromic temporalis muscle transfer. A preauricular incision was performed to expose the mandibular coronoid process. Using a saw, the coronoid process was transected. Three strips of the fascia lata were anchored to the muscle of the nasolabial fold through subcutaneous tunneling. The tension of the strips was adjusted by observing the shape of the nasolabial fold. When optimal tension was achieved, the temporalis muscle was sutured to the strips. The surgical results were assessed by comparing pre- and postoperative photographs. Three independent observers evaluated the photographs. Results The symmetry of the mouth corner was improved in the resting state, and movement of the oral commissure was enhanced in facial animation after surgery.

Conclusions The orthodromic transfer of temporalis muscle technique can produce prompt results by applying the natural temporalis muscle vector. This technique preserves residual facial nerve function in incomplete facial nerve palsy patients and produces satisfying cosmetic outcomes without malar muscle bulging, which often occurs in the turn-over technique.

Keywords Facial paralysis / Temporal muscle / Fascia lata

\author{
Correspondence: Dong Hee Kang \\ Department of Plastic and \\ Reconstructive Surgery, Dankook \\ University Hospital, Dankook \\ University College of Medicine, \\ 359 Manghyang-ro, Dongnam-gu, \\ Cheonan 330-715, Korea \\ Tel: +82-41-550-6477 \\ Fax: +82-41-554-6477 \\ E-mail:dhkcool@daum.net
}

This article was presented at the 69th Congress of the Korean Society of Plastic and Reconstructive Surgeons on November 11, 2011 in Seoul, Korea.

No potential conflict of interest relevant to this article was reported.

\section{INTRODUCTION}

Permanent facial palsy is considered to be a serious functional and aesthetic impairment. It deprives patients of a natural smile, which is one of the important means of mental and affective expressions.

The dynamic facial reanimation technique must produce a coordinated, spontaneous, and symmetrical smile. Among the various procedures for reconstructing facial paralysis, cross facial nerve grafting, following free muscle transfer, is the most effective surgical procedure for achieving symmetrical spontaneous motion for complete type paralysis patients [1]. However, it requires a much longer period, and the outcome is delayed for up to a year. The majority of patients with facial paralysis are the incomplete type. For those patients, reconstruction with a free muscle transfer might be an inordinate procedure. Moreover, 
the preservation of residual facial nerve function is also important for incomplete facial nerve palsy patients. Therefore, less invasive surgery that can show prompt results is required for those patients.

Reanimation of facial paralysis using the temporalis muscle turned over the zygomatic arch was used as early as 1934 by Gillies [2]. In this method, the temporalis muscle origin was elevated and flipped over above the zygomatic arch to the nasolabial fold. This has the disadvantages of donor site depression and midfacial widening. Furthermore, the temporalis muscle action vector was changed to an antidromic direction. In 1953, McLaughlin [3] modified the temporalis muscle transfer technique in an orthodromic manner, that is, without an inversion of the temporal muscle. This procedure avoids the fullness over the zygomatic arch area and the temporal donor site depression. It is surgically simple, and the vector of the transferred muscle action is similar to that of the original muscle direction. In this study, an attempt to improve the orthodromic temporalis muscle transfer method is reported. The improved method is more easily performed, requiring preauricular and nasolabial intermittent incisions with minimal dissection, and offers good aesthetic results in incomplete facial nerve palsy patients.

\section{METHODS}

\section{Patients}

Between August 2009 and November 2011, 6 patients who had suffered from unilateral incomplete facial nerve palsy underwent orthodromic temporalis muscle transfer. The ages of the $6 \mathrm{pa}-$ tients ranged from 29 to 66 years, with a mean of 48.2 years. One was male and 5 were female. The etiology of incomplete facial palsy included Bell's palsy $(n=3)$, parotid tumor $(n=1)$, penetrating injury $(n=1)$, and congenital origin $(n=1)$. The mean time between the onset of palsy and orthodromic temporalis transfer was 21.1 years (range, 1 to 51 years) (Table 1 ).

The surgical indications for this study were as follows: an extended period of paralysis (more than 1 year), no improvement in facial palsy symptoms for at least six months, and incomplete type. The patients wanted to improve the symmetry when they were resting and smiling.

\section{Surgical technique}

Under general anesthesia, the face and thigh are prepared. Through the preauricular crease, an approximately $6 \mathrm{~cm}$ incision is made. Through this incision, a minimal skin flap is raised as needed. Blunt dissection is performed to expose the mandibular coronoid process, which is insertion of the temporalis muscle, and right angle retractors are placed to protect the facial nerve. A drill is used to make a hole in the coronoid process, and then a wire is placed through the hole to prevent retraction. Using a reciprocating saw, the coronoid process is transected at the neck and is mobilized from the surrounding tissue along with the attached temporalis tendon, thus leaving as much of the temporalis tendon as possible.

Three intermittent $0.5 \mathrm{~cm}$ incisions are made in the new nasolabial crease. These incision points are determined before the operation by a preoperative smile analysis. This new crease should be located more medially than the original nasolabial fold, because the original crease will have already drifted laterally due to the loss of muscle tone. The three points should be located in the orbicularis oris muscle on the new nasolabial crease. To produce a natural smile, the vector of traction by the temporalis muscle is in a postero-lateral direction. Determining an appropriate traction point and traction vector is very important for a natural smile.

The fascia lata is harvested from a lateral thigh and divided into three strips, approximately $1 \mathrm{~cm} \times 14 \mathrm{~cm}$ in size. The strips are anchored to the orbicularis oris muscle at the key point through subcutaneous tunneling from the preauricular incision to the nasolabial crease (Fig. 1). Anchoring sutures placed too superficially into the muscle would result in lip eversion, whereas placement too deeply would result in puckering of the lip. Care should be taken with each strip not to twist or tether the tissue. Then, anchoring sutures to the nasolabial crease should be affixed as tight as possible, using the multiple buried suture

$$
\text { Table 1. Clinical data summary for study group }
$$

\begin{tabular}{|lclllccl|}
\hline Case & Age (yr)/Sex & Etiology & Affected side & Incision & $\begin{array}{c}\text { Duration of } \\
\text { palsy (yr) }\end{array}$ & Result (score) & Ancillary procedures \\
\hline 1 & $45 / \mathrm{M}$ & Bell's palsy & Right & Preauricular & 30 & 2.3 & Forehead lift \\
2 & $29 / \mathrm{F}$ & Bell's palsy & Left & Preauricular & 12 & 3.7 & Lower blepharoplasty \\
3 & $58 / F$ & Parotid tumor & Left & Preauricular & 3 & 2.7 & Forehead lift \\
4 & $40 / F$ & Bell's palsy & Left & Preauricular & 30 & 3.7 & Lower blepharoplasty \\
5 & $66 / F$ & Penetrating injury & Left & Previous scar & 1 & 3.0 & Lower blepharoplasty \\
6 & $51 / F$ & Congenital & Left & Preauricular & 51 & 3.7 & None \\
\hline a) poor; 2, fair; 3, good; 4, excellent. & & & & & \\
\hline
\end{tabular}




\section{Fig. 1. Schematic illustrations of surgical technique}

(A) Line of preauricular incision (blue line). (B) Transected coronoid process and insertion of temporalis muscle. (C) The strips of the fascia lata graft to the orbicularis oris muscle and temporalis muscle insertion.
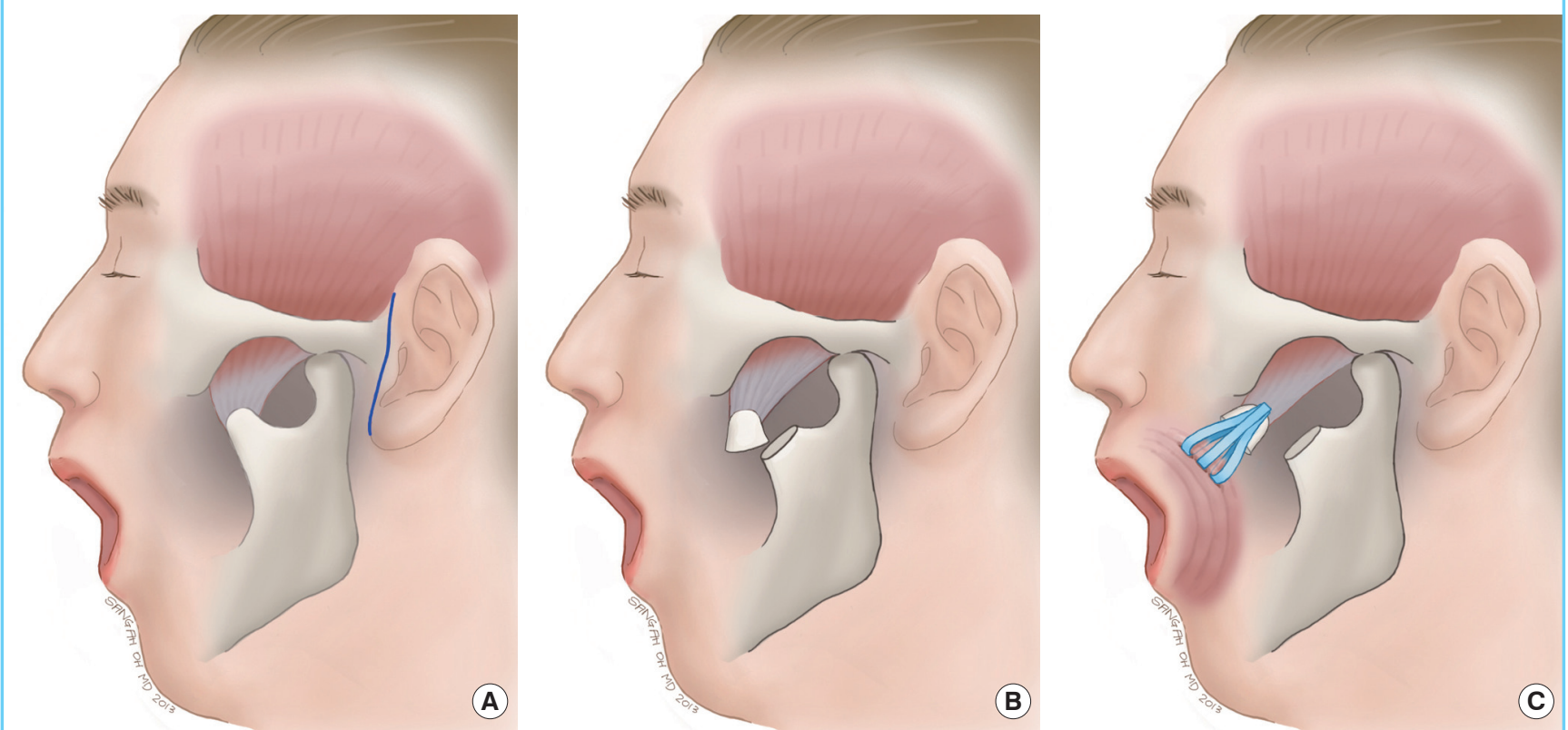

Fig. 2. Photographs taken during the temporalis transfer surgery

(A) Case 6. The red marking is the incision line and green area is the dissection area in which the coronoid process will be exposed. (B) Case 4. This case was approached through the preauricular incision. The transected coronoid process (black arrowhead) was held with a wire to prevent retraction. The strips of the fascia lata sutured in the mimic muscles of the nasolabial fold. Then, the surgeon adjusted the tension of the strips, pulling the fascia or releasing using the mosquito forceps (white arrows).
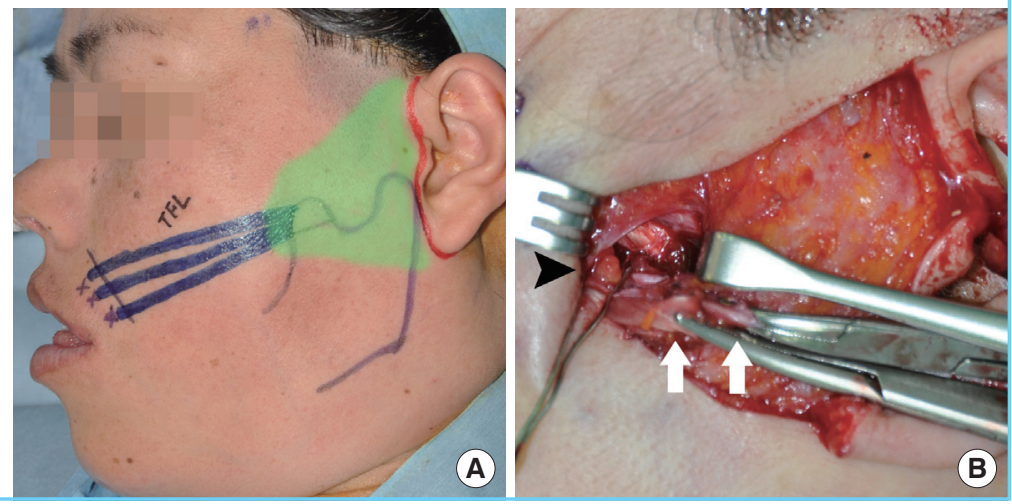

method with nylon 4-0. Afterwards, the tension of the strips is adjusted by observing the shape of the bilateral nasolabial fold (Fig. 2). The tension should be overcorrected due to loosening within a few weeks after surgery. When the optimal tension is achieved, the strips of the fascia lata are sutured, with nylon 3-0, to the tendinous portion of the temporalis muscle insertion.

Hemostasis should be checked before skin closure. Postoperatively, patients need mild compressive dressing above the dissection plane and have to be cautious when eating and talking.

\section{Ancillary procedure}

Some of the facial palsy patients suffered from ectropion or brow ptosis. In such cases, the authors performed correction of the ectropion or a forehead lift, as appropriate. Three patients underwent lower blepharoplasty with a fascia lata sling, and two patients underwent a forehead lift using Endotine forehead fixation device (Coapt Systems Inc., Palo Alto, CA, USA) (Table 1).

\section{Evaluation}

The authors evaluated the results six months after surgery. The postoperative follow-up ranged from 6 to 12 months. The surgical results were assessed by comparing the pre- and postoperative photographs (Figs. 3, 4). For evaluation of the results, the authors used the classification by May and Drucker [4]; 1, poor (the corner of the mouth on the paralyzed side drooped); 2 , fair (the corners of the mouth were symmetrical with the face in repose); 3 , good (discreet smile, pulling up the corner of the mouth); and 4, excellent (patient smiles voluntarily with exposure of the 


\section{Fig. 3. Preoperative and postoperative photographs of case 4}

A 40-year-old woman with incomplete left facial nerve palsy due to Bell's palsy. (A) Preoperative resting state photograph. (B) Seven months after the operation, at a resting state. (C) Preoperative smiling appearance. (D) Seven months after the operation, smiling appearance.
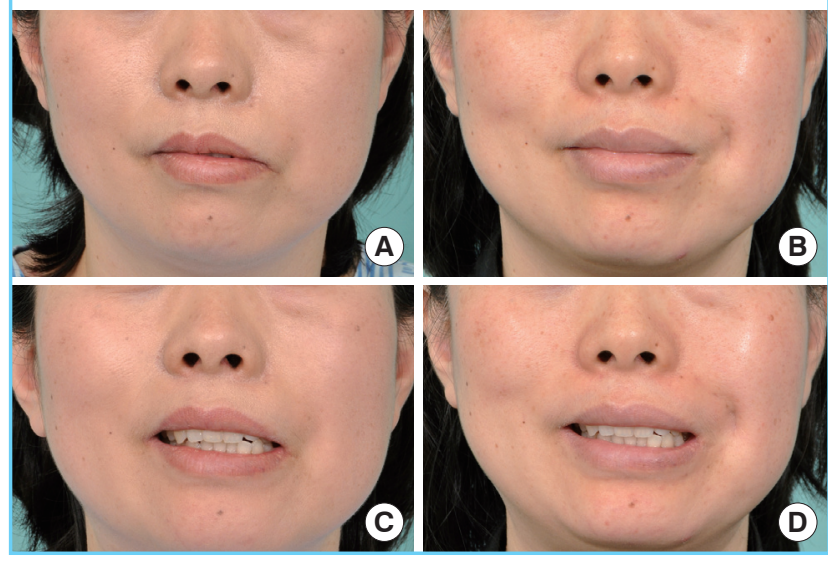

teeth). Three independent observers evaluated the photographs, and then the authors averaged their score.

\section{RESULTS}

All of the participants had the same preoperative score of 1 because the corner of the mouth on the paralyzed side drooped in all of the patients before surgery. The average postoperative score of all 6 cases was 3.2 (Table 1). The 6 patients underwent the orthodromic transfer of the temporalis muscle technique, improved their symmetry without any damage to the residual facial nerve function, and none developed procedure-induced complications, such as a facial contour defect, lip eversion or puckering, or skin tethering. The symmetry at rest and smile movement were improved, and the patients' satisfaction was high.

\section{DISCUSSION}

Reanimation of the paralyzed face has been one of the greatest challenges faced by surgeons for centuries. The purpose of facial palsy surgery is to reconstruct the natural and symmetric expression of the resting and animated face [4-8]. Despite the existence of many procedures for reconstructing the paralyzed face, there is no gold standard procedure that will fully return normal facial function $[9,10]$.

The temporalis muscle transfer technique to reanimate the face in facial nerve palsy was first described by Gillies [2]. He described turning down the temporalis muscle origin across the zygomatic arch with a tensor fasciae latae graft to the upper and lower lips. However, this technique leads to malar area

\section{Fig. 4. Short-term perioperative photographs of case 6}

A 51-year-old woman with congenital incomplete left facial nerve palsy. (A) Preoperative resting state photograph. (B) Two months after the operation, at a resting state. (C) Preoperative smiling appearance. (D) Two months after the operation, smiling appearance.
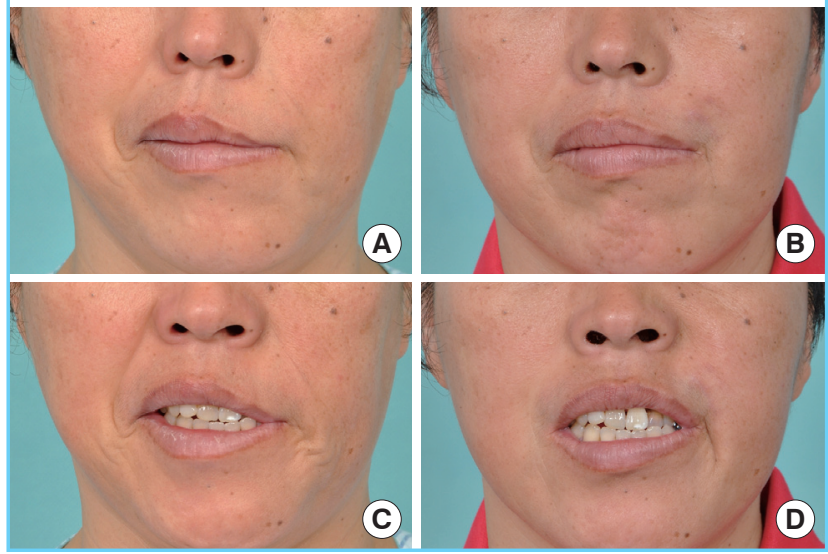

bulging and switches the vector of the temporalis muscle action direction. As opposed to the Gillies method, which transposed the origin of the temporalis muscle, McLaughlin [3] described transferring the insertion of the temporalis muscle in an orthodromic manner. Through an intraoral approach, the temporalis muscle was transferred inferiorly, by osteotomy of the coronoid process. The muscle was extended by a tensor fasciae latae graft anchored to the orbicularis oris muscle. This improved on the disadvantages of the Gillies method. However, an intraoral incision is inconvenient in approaching the coronoid process. Thus Breidahl et al. [7] modified this technique by using an external approach. Through a vertical incision in the temporal region, extending inferiorly into the level of the tragus, the zygomatic arch was resected to expose the coronoid process. The temporalis muscle insertion was detached from the coronoid process and anchored to the mouth corner with a fascia lata graft via a nasolabial incision.

Recently, Labbe and Huault [11] described a temporalis muscle lengthening technique without a fascia lata graft through the external approach [12-14]. The Breidahl and Labbe techniques both require performing ostectomy of the zygomatic arch to visualize the temporalis muscle and making a wide external incision with a broad dissection area. This leads to increasing the risk of damage to residual facial nerve function and denervation of the temporalis muscle.

Our study introduces a modification of the orthodromic temporalis muscle transfer technique without zygomatic arch resection for the reanimation of the paralyzed face. The antidromic temporalis muscle origin transfer technique adds bulkiness over the zygomatic arch and a depression in the temple, whereas the orthodromic temporalis transfer technique does not change the 
facial contour. This shows that a less invasive procedure can provide good results that are evident immediately and persist over the long term. In addition, it is a single stage technique, which is easily performed, and can be applied on patients of any age.

Our modification also uses only a $6 \mathrm{~cm}$ incision in the preauricular area and tiny intermittent incisions in the nasolabial fold, which are the anchoring points. All of the procedures can be performed through this small incision and minimal dissection. This technique is appropriate for use in patients with incomplete type facial paralysis because there is no danger of damaging any residual facial nerve function. Furthermore, patients fully utilize their nerve function.

In summary, orthodromic temporalis insertion transfer with fascia lata suspension is a safe and effective method of correcting functional and aesthetic asymmetries in incomplete facial nerve palsy patients. This technique is simple and shows good results immediately while preserving residual facial nerve function.

\section{REFERENCES}

1. Fattah A, Borschel GH, Manktelow RT, et al. Facial palsy and reconstruction. Plast Reconstr Surg 2012;129:340e$52 \mathrm{e}$.

2. Gillies H. Experiences with fascia lata grafts in the operative treatment of facial paralysis: (section of otology and section of laryngology). Proc R Soc Med 1934;27:1372-82.

3. McLaughlin CR. Surgical support in permanent facial paralysis. Plast Reconstr Surg (1946) 1953;11:302-14.

4. May M, Drucker C. Temporalis muscle for facial reanimation. A 13-year experience with 224 procedures. Arch Otolaryngol Head Neck Surg 1993;119:378-82.

5. Baker DC, Conley J. Regional muscle transposition for reha- bilitation of the paralyzed face. Clin Plast Surg 1979;6:31731.

6. Viterbo F, de Paula Faleiros HR. Orthodromic transposition of the temporal muscle for facial paralysis: made easy and better. J Craniofac Surg 2005; 16:306-9.

7. Breidahl AF, Morrison WA, Donato RR, et al. A modified surgical technique for temporalis transfer. Br J Plast Surg 1996;49:46-51.

8. Rubin LR. The anatomy of a smile: its importance in the treatment of facial paralysis. Plast Reconstr Surg 1974;53: 384-7.

9. Sidle DM, Fishman AJ. Modification of the orthodromic temporalis tendon transfer technique for reanimation of the paralyzed face. Otolaryngol Head Neck Surg 2011;145:1823.

10. Byrne PJ, Kim M, Boahene K, et al. Temporalis tendon transfer as part of a comprehensive approach to facial reanimation. Arch Facial Plast Surg 2007;9:234-41.

11. Labbe D, Huault M. Lengthening temporalis myoplasty and lip reanimation. Plast Reconstr Surg 2000;105:1289-97.

12. Har-Shai Y, Metanes I, Badarny S, et al. Lengthening temporalis myoplasty for facial palsy reanimation. Isr Med Assoc J 2007;9:123-4.

13. Har-Shai Y, Gil T, Metanes I, et al. Intraoperative muscle electrical stimulation for accurate positioning of the temporalis muscle tendon during dynamic, one-stage lengthening temporalis myoplasty for facial and lip reanimation. Plast Reconstr Surg 2010;126:118-25.

14. Nduka C, Hallam MJ, Labbe D. Refinements in smile reanimation: 10-year experience with the lengthening Temporalis Myoplasty. J Plast Reconstr Aesthet Surg 2012;65:851-6. 